\title{
Les néoruraux en cavale vers la campagne : quelles conséquences pour le Québec?
}

\author{
Laurie Guimond $^{\mathrm{a}}$, Myriam Simard ${ }^{\mathrm{b}}$
}

RÉSUMÉ. La migration vers les campagnes de populations d'origine urbaine, qu'on surnomme les néoruraux, s'effectue à l'échelle du Québec, autant dans les régions adjacentes aux grands et moyens centres urbains de la vallée laurentienne qu'ailleurs dans la province. Cet article s'intéresse à leur profil et aux motivations qui justifient la concrétisation de leur projet migratoire. En outre, il explore les conséquences multiformes de leur établissement à la campagne, tout en proposant certaines pistes d'action pour répondre aux défis sous-jacents au phénomène de néoruralité.

$\boldsymbol{A B S T R A C T}$. The migration to rural areas of urban populations, whom we call neo-rurals, occurs across Quebec in regions next to large and medium-sized urban centres of the Laurentian valley and elsewhere in the province. This article discusses their profile and the motivations driving their migration. It explores the multifaceted consequences of their settling in rural areas, all the while proposing certain courses of action to meet the underlying challenges of the neo-rurality phenomenon.

\section{Introduction}

Ce n'est plus un secret pour personne que les campagnes se transforment depuis plusieurs décennies, notamment par l'arrivée de nouvelles populations qu'on surnomme les néoruraux. Qui sont-ils? Quel âge ont-ils? Pourquoi quittent-ils la ville pour aller vivre à la campagne? Leur projet migratoire est-il définitif? Quelles sont les conséquences de leur installation pour le développement territorial? Quelles actions s'imposent pour répondre aux nouveaux défis posés par ce changement?

Au Québec, la migration d'urbains vers les campagnes s'est d'abord opérée avec le retour à la terre de certains hippies de la contreculture des décennies 1960 et 1970. Elle s'est ensuite accentuée avec l'établissement de néoruraux aux profils variés. Malheureusement, au Québec comme au Canada, aucune donnée statistique ne permet de dater, de chiffrer ni de décrire sommairement ce phénomène. Par contre, des indicateurs, comme celui du solde migratoire interrégional ${ }^{1}$, permettent de le déceler dans différentes municipalités régionales de comté (MRC), dont Portneuf, Maskinongé, le Val-
Saint-François, les Collines-de-l'Outaouais, les Îles-de-la-Madeleine, Avignon, Antoine-Labelle, Matawinie, Brome-Missisquoi et Arthabaska. Nos recherches, couplées à d'autres menées depuis plus de 15 ans dans des régions connaissant l'arrivée de ces nouveaux résidents, révèlent certaines grandes tendances à ce sujet.

\section{Qui? Où? Pourquoi? Comment? Tendances générales liées à la néoruralité}

Les néoruraux sont des individus qui vivaient en ville et qui ont fait le choix de s'établir en permanence en milieu rural ${ }^{2}$. Le seuil temporel généralement retenu au Québec et ailleurs dans le monde est celui de 20 ans puisque, au-delà de cette période, les néoruraux sont plutôt considérés comme des résidents de longue date. Il ne faut pas confondre les néoruraux avec les villégiateurs ou les touristes, vu que leur déménagement en campagne n'est ni passager ni saisonnier, tout comme ils diffèrent des banlieusards, qui habitent les banlieues et non les campagnes.

\footnotetext{
a Professeure agrégée, département de géographie, Université du Québec à Montréal

b Professeure honoraire, Institut national de la recherche scientifique
} 
La migration de la ville vers des territoires moins densément peuplés s'effectue à l'échelle du Québec, autant dans les régions adjacentes aux grands et moyens centres urbains méridionaux (Domon, Ruiz, Paquette et Roy, 2011; Roy, Paquette et Domon, 2013; Simard et Guimond, 2010) que dans les régions septentrionales (Guimond et Desmeules, 2019). Malgré les contrastes géographiques, culturels, sociodémographiques et économiques de la Belle Province, les défis liés à la néoruralité sont communs partout: attirer les nouveaux résidents en faisant connaitre ses atouts; accueillir en proposant des mesures multiformes pour encadrer l'établissement; cobabiter en valorisant la mixité par la rencontre et la collaboration de populations aux origines multiples; et retenir en prévoyant des mesures à long terme. Ces défis sont exacerbés par l'hétérogénéité des nouvelles populations rurales et de leurs motivations de s'implanter en campagne ainsi que par leurs modes d'habiter différenciés.

Les néoruraux font partie d'un groupe non homogène ayant des profils diversifiés : âge, origine géographique, statut familial, classe sociale, profession et revenus. On compte ainsi des jeunes pour qui cette installation à la campagne concorde avec la naissance des enfants, l'achat d'une propriété ou la concrétisation d'un projet occupationnel inédit; des adultes d'âge moyen qui optent pour la campagne afin de réaliser de nouveaux défis; ou des adultes d'âge mûr à la préretraite ou à la retraite en quête de quiétude. La migration ville-campagne se définit donc au gré des principales étapes de la vie.

Les motifs de migration sont tout aussi variés que les profils des néoruraux, mais ils sont intimement liés à la recherche d'une meilleure qualité de vie et d'un équilibre entre vie familiale, professionnelle et sociale. Une synthèse des facteurs d'attraction, d'insertion et de rétention dans l'espace rural québécois démontre bien qu'ils sont complexes et interreliés. En effet, de nombreuses considérations, aussi bien professionnelles et financières que personnelles, familiales, sociocommunautaires, culturelles et environnementales, interviennent simultanément dans la décision d'aller vivre dans ce type de milieu, d'y rester ou de le quitter (Simard, 2011).

Ces facteurs se découpent en quatre catégories, qui ne sont pas mutuellement exclusives (Simard et Guimond, 2009). D'abord, les raisons afférentes aux attraits géophysiques et sociaux de la campagne attirent les urbains: beaux paysages, proximité de la nature, rythme et style de vie, convivialité, sécurité, qualité de l'air, cadre idéal pour les loisirs extérieurs et un jardin, etc. Ensuite, des raisons d'ordre familial incitent plusieurs à faire le grand saut: élever les enfants, suivre le conjoint, rejoindre la famille et la parenté. Puis, des raisons professionnelles justifient ce déplacement : obtention d'un emploi, désir de devenir travailleur autonome ou de démarrer une entreprise. Enfin, des raisons économiques rattachées à la perception d'un coût de vie moindre, à des mesures incitatives salariales ou à l'accès à la propriété pèsent également dans la balance.

Ces deux derniers motifs rejoignent particulièrement les professionnels du secteur public (éducation, santé, services sociaux, sécurité publique, etc.) qui choisissent de vivre dans des communautés septentrionales autochtones et non autochtones, où les besoins en main-d'œuvre sont criants. Il s'agit d'infirmiers insatisfaits de leurs conditions de travail dans les hôpitaux des centres urbains (horaire surchargé, instabilité, roulement de personnel, méconnaissance et anonymat des patients, etc.) et désireux de s'épanouir professionnellement en offrant des soins de qualité et de proximité à leurs patients. Ils sont des enseignants lassés de faire de la suppléance et qui rêvent d'avoir leur "propre classe ». Ils sont de jeunes adultes à leur entrée sur le marché de l'emploi qui décident de s'éloigner des grands centres pour acquérir une expérience professionnelle solide et polyvalente, avec le lot de défis que l'éloignement comporte. Ils sont aussi des professionnels dans la cinquantaine ayant connu des discriminations d'embauche liées à leur âge ailleurs au Québec qui souhaitent terminer leur carrière dans le Nord. En somme, les nouveaux résidents du Nord sont surtout des actifs à la recherche d'une stabilité leur permettant de s'accomplir professionnellement, tout en ayant accès à des conditions de travail et à un salaire intéressants, puis à un milieu de vie attrayant (petitesse des communautés, proximité de la nature, nordicité, exotisme, vie quotidienne en milieu autochtone, etc.) (Guimond et Desmeules, 2019).

Plus largement, cette migration est loin d'être immuable et irréversible, car elle est marquée par divers allers-retours entre la ville et la campagne, selon deux échelles spatiotemporelles. Premièrement, dans la vie quotidienne, les visites des amis et de la famille en ville, les activités urbaines sportives, récréatives ou culturelles, le magasinage, le 
désir de l'effervescence urbaine et les liens professionnels sont autant de raisons de fréquenter régulièrement l'ancien lieu de résidence. Certains néoruraux gardent un pied-à-terre en ville (condo, appartement, chambre chez des amis ou parenté) dans l'optique de faciliter leurs séjours urbains. Deuxièmement, au sein même du projet migratoire, les retours en ville peuvent s'effectuer selon des évènements décisifs de la vie : études secondaires et postsecondaires des enfants, divorce ou décès d'un conjoint, rapprochement des enfants et des petits-enfants ou accès à des soins de santé.

Ainsi, l'absence de rupture définitive des néoruraux avec la ville se constate. Une complémentarité rurale-urbaine apparait puisque les deux milieux sont sujets à des va-et-vient constants et à des migrations plurielles selon les étapes de la vie (Simard, 2016). Par conséquent, des échanges, influences réciproques et interconnexions étroites caractérisent ces rapports ville-campagne.

\section{Incidences de la néoruralité sur le développement territorial}

L'arrivée d'urbains entraine inévitablement des transformations majeures dans les campagnes. Ceux-ci apportent une contribution favorable en stimulant un renouvellement de la population et, conséquemment, un maintien des services publics et de proximité comme les écoles, les garderies, la poste et les commerces. Ils engendrent une diversification d'activités par leurs demandes, la création d'entreprises et l'essor de la consommation locale (entretien et rénovation des propriétés, alimentation, loisirs). Généralement soucieux de dynamiser leur nouveau milieu de vie, les néoruraux n'hésitent pas à s'impliquer localement, à partager leurs expertises et à mettre à profit leurs expériences antérieures et leurs réseaux. Ils participent à la consolidation des secteurs artistique et culturel par des initiatives et par l'émergence de projets communautaires novateurs. Par leur engagement sur les plans politique et environnemental, ils valorisent la conservation du patrimoine bâti et naturel, tout en développant une sensibilisation accrue aux qualités et richesses écologiques du territoire rural (ex. : protection des espaces verts et des paysages).

La présence des néoruraux peut toutefois susciter des réactions défavorables tant de la population d'accueil que de certains néoruraux, surtout face aux réflexes urbains (ex. : demandes excessives en équipements et services). La cohabitation peut être parfois difficile entre les divers ruraux, anciens comme nouveaux (Guimond, Gilbert et Simard, 2014). Le fait qu'ils ne sont pas natifs du même univers et qu'ils ont un passé et des expériences de vie distincts contribue à les éloigner. Cette distance est créée, d'une part, par le passé ancestral et le patrimoine familial local qui unissent les ruraux de longue date et, d'autre part, par le statut d'étranger des nouveaux résidents. Ainsi, une certaine réserve mutuelle, renforcée par la méconnaissance de l'autre et par des partis pris, rend difficile la pénétration de réseaux intergroupes.

Des pratiques et des représentations divergentes de la campagne (productive, résidentielle, récréative et de protection) sont alors des marqueurs de distance et des sources de conflits. Ces hostilités éclatent souvent à propos d'activités susceptibles de menacer la quiétude et le cadre champêtre recherchés par les exurbains, par exemple la production agricole avec ses « nuisances » olfactives, sonores et visuelles ou encore des projets industriels dans des zones fragiles ou protégées (Simard, 2017). Les décideurs locaux seront à l'avenir interpellés fréquemment par ces défis tant de cohabitation sereine entre des populations contrastées que de gouvernance équitable, transparente et progressiste en vue du bien-être de tous les citoyens.

Un clivage de classes est aussi noté dans plusieurs régions prisées pour leurs paysages attrayants, phénomène étroitement lié à celui de l'embourgeoisement rural (Simard et Guimond, 2012), alimenté également par la villégiature (Loyer et Doyon, 2019). Le Québec rural n'y échappe pas. Maintes régions accusent ainsi des hausses de taxes foncières (maisons, terrains et loyers), lesquelles entrainent l'exclusion de populations moins nanties et un sentiment de dépossession du territoire. Dès lors, des rapports de domination peuvent se créer, les individus ne partageant pas forcément les mêmes conditions sociales, notamment les revenus, les réseaux, les niveaux de scolarité, les catégories socioprofessionnelles, les expertises d'argumentation en public, etc. Cet embourgeoisement constitue certainement un des principaux défis associés à la transformation actuelle des campagnes.

Il va sans dire que la migration des néoruraux implique des rapports de pouvoir dans les interactions locales qui influent immanquablement sur la restructuration rurale (Simard, Guimond et Vézina, 2018). L'ensemble de ces incidences sous-entend tant un apport dynamique de ces populations néorurales sur 
le développement territorial que la nécessité de mieux arrimer certaines politiques à leur égard pour faciliter leur insertion globale de façon harmonieuse.

\section{Pistes de réflexion et d'action}

Pour que les campagnes tirent profit au maximum de ce phénomène migratoire perçu comme une solution de renouvellement de la population rurale, certaines actions s'imposent pour relever les nouveaux défis. Elles concernent prioritairement les facteurs d'attraction, d'accueil et de rétention des néoruraux; une cohabitation bonifiée par une lutte contre les préjugés, les exclusions et les discriminations de part et d'autre; ainsi qu'une gouvernance locale cohérente et solidaire (Simard, 2018). Ces pistes d'action se déploient de façon très inégale sur l'ensemble du territoire québécois. Elles sont concrétisées dans certaines municipalités, ou en cours d'élaboration, embryonnaires ou encore inexistantes dans d'autres.

D'abord, des politiques d'accueil personnalisé des néoruraux dans chaque municipalité (fête de bienvenue, trousse d'information, bons d'achat pour les produits locaux, rôle municipal de pivot de l'intégration, jumelage d'anciens et de nouveaux villageois) sont indispensables pour assurer un accueil cordial et efficace. À titre d'exemple probant, le Projet d'accueil et d'intégration solidaire (PAIS) de la MRC de Bécancour a remporté plusieurs prix pour son caractère innovant et pour sa vision à long terme de l'accueil et de l'intégration globale des nouveaux résidents sur son territoire. L'implication des populations déjà établies est au cœur de la démarche du PAIS, incitant ainsi une meilleure interconnaissance de populations aux origines différenciées.

À ces politiques d'accueil doivent se coupler des politiques familiales pour toutes les tranches d'âges, comme l'encouragent des programmes de soutien aux politiques familiales municipales offerts par le ministère de la Famille du Québec. L'entretien et le renouvellement des équipements municipaux principalement pour les jeunes familles devraient garantir des services de base et de qualité pour tous. L'accessibilité à des réseaux désormais essentiels comme Internet haute vitesse et la téléphonie mobile agit quant à elle sur l'attraction et sur la rétention des populations rurales, nouvelles comme anciennes.
En outre, des politiques et des actions pour contrer les effets pervers de l'embourgeoisement rural, dont les politiques résidentielles pour faciliter l'accès à la propriété des jeunes et des moins nantis, sont des actions auxquelles ont recours diverses municipalités : congé de droit de mutation (taxes de bienvenue), réduction des taxes municipales, offre de terrains, construction de logements accessibles, etc. Les projets de fermettes de la Coopérative du Rang 13 portés par des citoyens et des organismes de Saint-Camille en Estrie ont permis le déploiement de 25 fermettes, attirant ainsi autant de jeunes familles. Cette initiative fut un moyen de se prémunir contre la fermeture de l'école primaire. À Sutton, un projet de 18 logements abordables vise à faciliter l'installation de jeunes familles dans cette municipalité où l'embourgeoisement rural, le vieillissement de la population et le manque criant de main-d'œuvre engendrent des défis de taille, notamment en matière d'accès à un logis (Demers, 2019; voir Annexe).

Enfin, pour promouvoir une meilleure cohabitation, les populations rurales doivent miser sur la connaissance et la reconnaissance de l'autre, sur l'abolition des discriminations de tous types et sur le rejet des idées préconçues. À ce propos, il est impératif que tous les acteurs dépassent la vision traditionnelle de clivage entre les néoruraux et les ruraux de longue date, car des solidarités mutuelles et des complicités peuvent émerger de leurs rencontres, notamment lors de leur implication conjointe dans des dossiers environnementaux, économiques, sociaux et culturels. Par exemple, on voit de plus en plus de rapprochements intergénérationnels dans certains villages québécois, lors des fêtes villageoises ou lors d'initiatives de jumelage professionnel entre retraités et jeunes, rapprochements qui stimulent les échanges sur les expériences acquises et les contacts, peu importe leur origine urbaine ou rurale.

Sur le plan de la gouvernance municipale, une cohabitation réussie passe également par une sensibilisation des élus sur leurs responsabilités accrues d'accueil des nouvelles populations et sur l'exigence de surmonter les querelles intestines pour instaurer des mesures novatrices répondant à l'intérêt général de l'ensemble des habitants, nouveaux et anciens. Il faut retenir toutefois, à l'instar de municipalités comme Saint-Camille et Frelighsburg, qu'une cohabitation solidaire prend du temps. Elle connait généralement une période transitoire d'apprivoisement mutuel pouvant durer plusieurs 
années, à la faveur de projets communautaires concrets et de mobilisations citoyennes dynamiques où s'expérimente graduellement le vivre ensemble.

Afin que ces actions se matérialisent, il y a nécessité au premier chef de les mettre à l'ordre du jour des agendas de tous les acteurs concernés : élus, dirigeants d'organisme, villageois, associations, etc. Il importe alors d'adopter une vision à long terme, audelà de la seule étape du marketing territorial. Ensuite, une véritable concertation et une synergie proactive tant locales que régionales et provinciales sont nécessaires pour les réaliser pleinement. À ce titre, des municipalités, MRC, organisations et groupes de citoyens ont entrepris des initiatives fructueuses pour attirer de nouveaux résidents dans les milieux ruraux et pour faciliter leur insertion durable, et ce, à l'échelle de la province. Il est crucial cependant que tous aient accès à des ressources financières adéquates pour amorcer ou poursuivre de tels projets, sinon il y a danger de voir s'accentuer les conséquences perverses d'exclusion et la fragmentation de la société rurale.

\section{Conclusion}

La migration d'urbains vers la campagne n'est pas un évènement passager et éphémère, compte tenu de l'arrivée de la génération de baby-boomers à la retraite, de la quête constante d'une meilleure qualité de vie, de l'intensification du mouvement environnemental ainsi que d'un désir de quitter la ville et ses insécurités. Il est essentiel de briser le mythe selon lequel ces néoruraux ne suscitent que des problèmes ou des conflits dans les collectivités rurales, car ils contribuent manifestement à leur essor. En choisissant de vivre à la campagne et en s'investissant dans leur milieu, ils assurent un renouvellement démographique qui permet de maintenir des services de proximité et de créer des activités variées.

Force est d'admettre que les néoruraux sont des acteurs indéniables du développement territorial. Leur présence même, qui se négocie et se concilie avec celle des populations de longue date, réaffirme le dynamisme des campagnes et rappelle la volonté et l'importance d'occuper l'ensemble du territoire québécois, au-delà des frontières des villes.

Les fortes mobilités qui caractérisent les modes de vie contemporains obligent néanmoins à réfléchir au statut incertain et instable de la notion de «permanence » au $21^{\mathrm{e}}$ siècle, car la plurimobilité et la migration non permanente existent et doivent être considérées comme des phénomènes normaux et temporaires rattachés aux différentes étapes de la vie, et non comme des échecs de rétention. De la même manière que le démontrent les résultats de nos recherches, un départ en ville des néoruraux pour la scolarisation avancée de leurs enfants ne signifie pas qu'ils ne feront pas de retour à la campagne à un stade de vie ultérieur, surtout s'ils y ont été bien accueillis et intégrés. Les décideurs doivent alors s'assurer que les politiques appropriées soient implantées pour les attirer à nouveau et assurer une mixité sociale cohésive dans les villages, indépendamment de l'origine rurale ou urbaine des résidents.

\section{NOTES}

1 Le solde migratoire réfère à la différence entre les entrées et les sorties des migrants infraprovinciaux, interprovinciaux ou internationaux d'un territoire donné (ex. : région administrative, MRC, municipalité). L'Institut de la statistique du Québec comptabilise les soldes migratoires à l'échelle de la MRC. Ainsi, un solde migratoire positif signifie qu'au terme d'une période, le nombre d'entrées dans une MRC donnée est supérieur au nombre de sorties. Un solde migratoire négatif signifie l'inverse. Il faut demeurer prudent lors de l'utilisation de ces données statistiques. Par exemple, même si un solde migratoire est négatif, ce qui laisse sousentendre un exode rural, une migration à contresens peut s'opérer, témoignant d'une migration de la ville vers les campagnes.

2 La définition de milieu rural que nous retenons s'appuie sur la typologie de Statistique Canada, laquelle comprend tout le territoire situé à l'extérieur des agglomérations de recensement (10 000 habitants ou plus) et des régions métropolitaines de recensement (100 000 habitants ou plus) (Du Plessis, Beshiri, Bollman et Clemenson, 2002). 


\section{RÉFÉRENCES}

Demers, X. (2019, 6 mars). Première étape franchie pour un projet de 18 unités de logement abordables à Sutton. Journal Le Guide.com, $37(50)$, p. 3.

Domon, G., Ruiz, J., Paquette, S. et Roy, L. (2011). La recomposition sociodémographique des campagnes québécoises à la croisée des dynamiques agricoles et paysagères. Revue canadienne des sciences régionales/Canadian Journal of Regional Science, 34(4), 115-133.

Du Plessis V., Beshiri, R., Bollman, R. D. et Clemenson, H. (2002). Définitions de «rural» : série de documents de travail sur l'agriculture et le milieu rural. Document de travail no 061. Ottawa, ON : Statistique Canada. Repéré à www150.statcan.gc.ca/n1/fr/pub/21-601$\mathrm{m} / 2002061 / 4241273$-fra.pdf?st=urtoL16z

Guimond, L. et Desmeules, A. (2019). Choosing the northern periphery: Paradoxes in the ways of dwelling of new residents of Eastern Minganie (North Shore, Québec, Canada). Population, Space and Place, 25(6). https://doi.org/10.1002/psp.2226

Guimond, L., Gilbert, A. et Simard, M. (2014). Faire sa place et être de la place : la rencontre timide des nouveaux ruraux et des populations plus anciennes au Québec. Le géographe canadien/The Canadian Geographer, 58(1), 123-139. https://doi.org/10.1111/j.1541-0064.2013.12051.x

Loyer, F. et Doyon, M. (2019). L'appropriation des terres agricoles à des fins de villégiature : le cas de la municipalité régionale de comté de Memphrémagog. Recherches sociographiques, LX(1), 167-195.

Roy, L., Paquette, S. et Domon, G. (2013). Anciens et néoruraux : préjugés, tensions et affinités au sein d'une localité rurale québécoise. Géographie, économie, société, 15(1), 67-88. https://doi.org/10.3166/ges.15.67-88

Simard, M. (2011). Quand la famille pèse dans la balance... lors de la décision de venir vivre en milieu rural ou de le quitter. Enfances, Familles, Générations, 15, 131-157. https://doi.org/10.7202/1008149ar

Simard, M. (2016). Prendre la clef des champs en famille pour vivre dans la campagne québécoise rime-t-il avec un abandon de la ville? Bulletin de liaison du Partenariat Familles en mowvance et dynamiques intergénérationnelles, 14, 4-8.

Repéré à http://espace.inrs.ca/id/eprint/6807

Simard, M. (2017). Environnement, conflits et tractations entre divers acteurs ruraux au Québec : une mobilisation inégale du capital environnemental? Norois, 243(2), 111-126. Repéré à www.cairn.info/revue-norois-2017-2-page-111.htm

Simard, M. (2018). La migration de la ville à la campagne des citadins et les défis de cohabitation, d'embourgeoisement rural et de gouvernance. Dans F. Huot et S. Seguin-Lamarche (dir.), L'état du Québec 2019 (p. 288-294). Montréal, QC : Institut du Nouveau Monde/Del Busso.

Simard, M. et Guimond L. (2009). L'hétérogénéité des nouvelles populations rurales : comparaison dans deux MRC contrastées au Québec. Recherches sociographiques, 50(3), 475-505. https://doi.org/10.7202/039064ar

Simard, M. et Guimond, L. (2010). La migration de la ville vers la campagne au Québec? Portrait sociodémographique et économique de deux MRC contrastées et de leurs nouveaux résidents. Dans P. Cambon et S. Ladouceur (dir.), Panorama des régions du Québec: édition 2010 (p. 13-29). Québec, QC : Institut de la statistique du Québec. Repéré à www.stat.gouv.qc.ca/statistiques/profils/ panorama-regions-2010.pdf

Simard, M. et Guimond L. (2012). Que penser de l'embourgeoisement rural au Québec? Visions différenciées d'acteurs locaux. Recherches sociographiques, 53(3), 527-553. https://doi.org/10.7202/1013408ar

Simard, M., Guimond, L. et Vézina, J. (2018). Alliances et tensions entre néoruraux et décideurs locaux dans le Québec rural. Revne Gouvernance, 15(2), 50-85. https://doi.org/10.7202/1058088ar 


\section{ANNEXE}

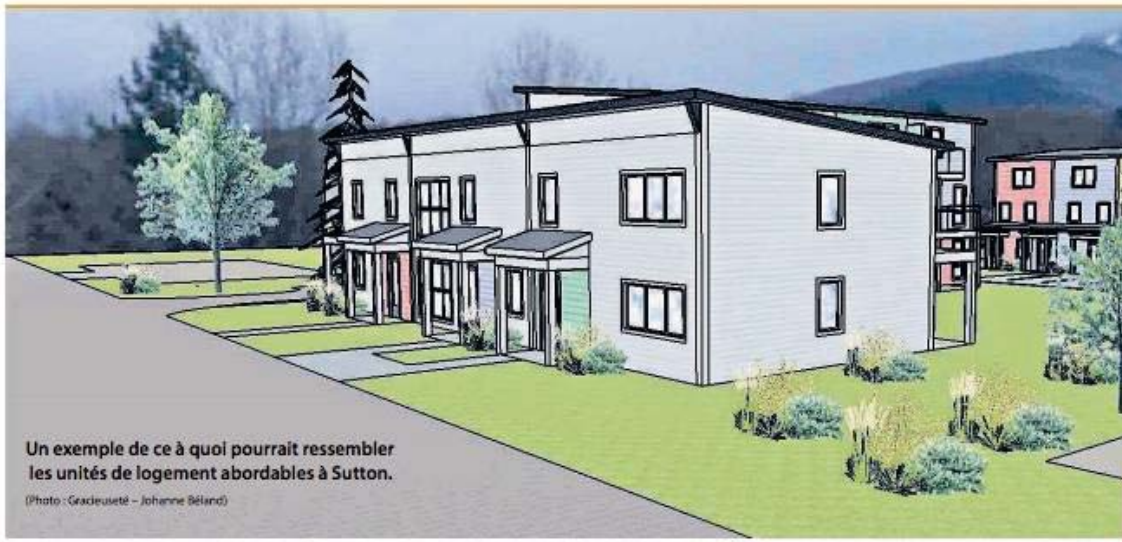

\section{Accès au logement}

Première étape franchie pour un projet de 18 unités de logement abordables à Sutton

\begin{abstract}
jusque dans les écoles. Selon des données des ce sens-là qu'on pousse le logement abordable. XAVIER DEMERS

HABITATION. Après près de dix ans à avoir Eastern Townships, la clientèle scolaire a dimi- prend ce virage-là et on ralentit ou on pourrait nué de $24,1 \%$ depuis 2001 a Sutton. $\quad$ en subir les conséquences. C'est le bon

travaillé sur le dossier, le projet des diminution, ça n'aide pas, mais c'est surtout Malgré les changements délus municipaux Habitations abordables Sutton continue à pour la revitalisation de la population et à Sutton, le projet a toujours bénéficié d'un avancer. Le projet, qui consiste à construire répondre aux besoins de nos commerçants, a appui de la part des différents conselils tout au 18 unités de logement abordables à Sutton, indiqué Kenneth Hill. Les gens qui vivent à l'ex- long de la réalisation du projet.

a franchi une première étape névralgique: tórieur et quitravaillenticićestcertainque tot celle de la présentation publique.

térieur et qui travaillent ici, c'est certain que, tốt ou tard, ils finissent par quitter. Quand ils PROJET quittent, bien souvent, la famille au complet

Le projet, dont la valeur est estimée entre «Les Habitations abordables Sutton, cest une suit. C'est un effet domino qu'll faut essayer d'ar- 2 MS et $2,5 \mathrm{MS}$, sera situé sur la rue Principale, organisation dont lobjectif est la construction rêter. Ce n'est pas la construction de 18 unités de près du CPE La Passerelle. Sur les 18 habitations, d'immeuble pour accueillir de jeunes familles à logement qui va faire ça, mais c'est un début. II jusqu’à neuf ménages pourront avoir droit à une Sutton, a expliqué d'entrée de jeu le président du faut y mettre un frein.» subvention, qui réduira le coût du loyer à $25 \%$ conseil d'administrationd'Habitations abordables Le problème d'attraction de main-d'oeuvre de leur revenu.

Sutton, Kenneth Hill. On a fait un sondage pour est d'autant plus important avec la difficulté "Les neuf autres seront également baissé à s'assurer qưon ne soit pas dans le champ avec d'accès au logement. Selon Habitations abor- $95 \%$ du tarif des loyers de la régions, a ajouté notre projet et il y a plus de 40 familles qui ont dables Sutton, la communauté d'affaires de la l'agent de développement d'Entraide Habitat répondu en avoir besoin. Une chose qui est ville voit d'un bon œeil limplantation d'un tel Estrie, Yves Perrier.

flagrante dans notre communauté, c'est quil faut projet.

Avec l'adoption de la loi 122, ce type de progarder nos baby-boomers, mais aussi nos jeunes «Les jeunes apportent dans la communauté jet ne sera pas soumis à l'approbation référenfamilles. Cest l'avenir de notre municipalité.s un dynamisme incroyable, a affirmé de son cóté daire. Si tout se passe bien, les unités d'habita-

Limpact du vieillissement de la population un des administrateurs d'Habitations abor- tion pourraient accueillir leurs premiers et de la gentrification de Sutton se fait ressentir dables Sutton, Gilles Lavoie. C'est un peu dans locataires d'íci l'automne 2020.
\end{abstract}

